\title{
Shared and specific patterns of structural and functional thalamo-frontal disturbances in manic and euthymic pediatric bipolar disorder
}

\author{
Yi-Bing Guo ${ }^{1,2} \cdot$ Wei-Jia Gao ${ }^{3} \cdot$ Zhi-Liang Long ${ }^{1} \cdot$ Wei-Fang Cao ${ }^{4} \cdot$ Dong Cui $^{5} \cdot$ Yong-Xin Guo ${ }^{4} \cdot$ Qing Jiao $^{4}\left(\right.$. $^{2}$ \\ Jian-Feng Qiu $\cdot{ }^{4}$ Lin-Yan Su ${ }^{6}$. Guang-Ming Lu ${ }^{7}$
}

Accepted: 7 August 2021 / Published online: 25 August 2021

(C) The Author(s) 2021

\begin{abstract}
Bipolar disorder (BD) is clinically defined by alternating depressive and manic episodes with a separated period of euthymia. Thalamo-frontal loop plays vital role in psychotic symptoms, altered motor control and executive difficulties in BD. It remains unclear that structural and functional alterations of thalamo-frontal loop among the different mood states in BD, especially in pediatric $\mathrm{BD}(\mathrm{PBD})$.

Twenty manic PBD (mPBD), 20 euthymic PBD (ePBD) and 19 healthy controls (HCs) were included in the study. By analyzing the T1 images and fMRI signals, thalamus volume and frontal grey matter cortical thickness were tested, and functional connectivity (FC) between bilateral thalamus and frontal cortex was calculated. Relationship between clinical indices and thalamo-frontal FC was also evaluated in $\mathrm{mPBD}$ and $\mathrm{ePBD}$ adolescents.

Compared to HCs, the cortical thickness of left middle frontal gyrus (MFG), bilateral superior frontal gyrus (SFG) was significantly decreased in both mPBD and ePBD patients, and volume of left thalamus and cortical thickness of right MFG significantly decreased in mPBD patients. Compared to that of the HCs and ePBD subjects, thalamo-frontal hyperconnectivity with MFG was found in mPBD, and compared with that of HCs, thalamo-frontal hypoconnectivity with precentral gyrus/SFG was found in ePBD. In ePBD patients, episode times positively correlated with FC values between thalamus and precentral gyrus.

The findings of the present study demonstrate detailed knowledge regarding shared and specific structural and functional disruption in thalamo-frontal loop in $\mathrm{mPBD}$ and ePBD subjects. Thalamo-frontal abnormalities reported in adult BD subjects were also observed in adolescent BD patients, and thalamo-frontal dysfunction may be a crucial treatment target in BD.
\end{abstract}

Keywords Thalamo-frontal loop · Manic pediatric Bipolar disorder · Euthymic pediatric bipolar disorder · Functional connectivity $\cdot$ Functional magnetic resonance imaging

Qing Jiao

bingbao17@163.com

1 Faculty of Psychology, Southwest University, Chongqing 400715, China

2 Research School of Psychology, The Australian National University, Canberra, Australia

3 Department of Child Psychology, The Children's Hospital, Zhejiang University School of Medicine, Hangzhou 310003, Zhejiang, China

4 Department of Radiology, Shandong First Medical University \&Shandong Academy of Medical Sciences, Tai' an 271016, Shandong Province, China
5 Institute of Biomedical Engineering, Chinese Academy of Medical Science \& Peking Union Medical College, Tianjin 300192, China

6 Mental Health Institute of the Second Xiangya Hospital, Central South University, Changsha 410011, China

7 Department of Medical Imaging, Jinling Hospital, Clinical School of Medical College, Nanjing University, Nanjing 210002, China 


\section{Introduction}

Bipolar disorder is a psychiatric disorder characterized by severe intermittent mood disorders that vary between mania, depression, and euthymia(Altinay et al., 2016), affected about 3.9\% of youth under age of 18(Van Meter, Moreira, \& Youngstrom, 2019). Corticolimbic model, including ventrolateral circuit, ventromedial circuit, dorsolateral circuit and anterior cingulate circuit, had been put forward to explain internal and external emotional control along with cognition in bipolar disorder(Brooks \& Vizueta, 2014). In this model, thalamus play a critical role through its bilateral projections with prefrontal cortex. Substantial evidence had elucidated that thalamus contributes to the pathogenesis of bipolar disorder(Duarte et al., 2016; Nery, Monkul, \& Lafer, 2013). However, inconsistent results had been reported showing increased, decreased and no different volume and functional connectivity (FC) in thalamo-frontal loop in BD subjects.

Several studies suggested volumetric deficits of the thalamus in BD patients. For example, decreased grey matter volume in right thalamus and bilateral prefrontal cortex were reported in BD subjects(Hibar et al., 2018; Yu et al., 2019). Increased left thalamic volume in male manic $\mathrm{BD}$ was found compared to healthy controls $(\mathrm{HCs})(\mathrm{Z}$. Chen et al., 2012). While patients with bipolar I disorder were found having no significant volumetric thalamus abnormalities during early stage of the disease(Arumugham et al., 2017). And no significant differences concerning the thalamus volume both in adult BD(L. Chen et al., 2018; Liberg et al., 2015) and pediatric BD(PBD) patients(Frazier et al., 2005) were also reported. On the context of frontal cortex, diminished grey matter of the prefrontal cortex in $\mathrm{BD}$ (Soloff et al., 2008) and larger grey matter volume of right inferior frontal gyrus in healthy offspring of $\mathrm{BD}$ parents(Hajek et al., 2013) had been reported. Disruptions of the structure may lead to dysfunction of the thalamofrontal loop, which may present as common symptom of $\mathrm{BD}$, such as failure in emotional regulation, altered motor control and executive difficulties. For instance, increased thalamic connectivity with orbitofrontal cortex has been demonstrated in BD adolescents(Ramsay, 2019), and in medication-free adolescents combining $\mathrm{BD}$ and attention deficit hyperactivity disorder(Son et al., 2017). In brief, accumulating evidence supported the structural and functional abnormalities of thalamo-frontal loop in $\mathrm{BD}$ patients. However, little attention has been paid to the structural and functional changes of thalamo-frontal loop among different mood states in BD, especially in pediatric $\mathrm{BD}$. In general, compared with that of adult BD, PBD presents with more atypical symptoms and comorbidities, difficult to complete remission, more recurrences and longer disease durations(Goldstein \& Birmaher, 2012). Investigation of thalamus and frontal cortex networks in manic PBD (mPBD) and eythymic PBD(ePBD) may be crucial for understanding the pathophysiology of PBD.

The aim of the current study is to explore the shared and specified structural and functional alterations of thalamofrontal loop in mPBD and ePBD. Based on previous findings, we predicted that both euthymia and manic patients with PBD would present abnormalities in thalamic and frontal structure and thalamo-frontal FC. While testing this hypothesis, the issue of thalamus volume and frontal grey matter cortical thickness were tested and FC between bilateral thalamus and frontal cortex was calculated. Relationship between clinical indices and thalamo-frontal FC was also evaluated in mPBD and ePBD subjects.

\section{Methods}

\section{Subjects}

The data of the present study was extracted from January 2012 to July 2014. Forty PBD patients (20 manic PBD and 20 euthymic PBD) were included in the present study, who were recruited from the Child and Adolescent Psychiatry Clinic of the Second Xiangya Hospital of Central South University in Changsha, Hunan. Nineteen age- and gendermatched healthy controls were recruited from local middle schools.

The criteria for the inclusion of PBD patients were as follows: 1) meeting Diagnostic and Statistical Manual for Mental Disorders, Fourth Edition (DSM-IV) criteria for bipolar disorder (BD), including hypomania ( $\geq 4$ days) or mania ( $\geq 7$ days) that met at least one full DSM-IV-TR criteria; 2 ) may be instructed to hold body still during MRI scanning; 3) Han nationality; 4) 10-18 years old; 5) right handedness. Exclusion criteria for all participants included: 1) IQ $<80$; 2) with alcohol or drug abusing or dependence; 3 ) with other mental disorders, such as schizophrenia or autism; 4) with major sensor motor handicaps. 5) with contraindications to MRI scans, including claustrophobia, stents, and metal implants.

All procedures in the present study were in accordance with the ethical standards of Xiangya Hospital and the National Research Council. Written informed consents were obtained from all the subjects and their guardian.

\section{Clinical evaluations}

By employing Schedule for Affective Disorders and Schizophrenia for School aged Children Present and Lifetime Versions (K-SADS-PL)(Kaufman et al, 1997), two experienced pediatric psychiatrists determined the diagnosis of all PBD 
patients. Criteria for diagnosis of manic PBD patients were DSM-IV criteria for mania or hypomania but were not met DSM-IV criteria for depressive symptom. Meanwhile, euthymic PBD subjects were not met DSM-IV criteria for mania, depression, hypomania or dysthymia, and should be in remission state for at least four weeks. All participants' intelligence was assessed by the Wechsler Abbreviated Scale of Intelligence (WASI). The severity of emotional symptoms of all of the subjects were assessed using Mood and Feelings Questionnaire (MFQ)(Chang et al., 2005) and Young Mania Rating Scale (YMRS)(Young et al., 1978) prior to MRI scanning. MFQ has 33 items, which is a well-designed measure to evaluate emotional symptoms of subjects. People with more severe of depression have a higher score of MFQ. YMRS has 11 items and is a clinician rating scale. YMRS scores were associated with manic symptoms, with higher scores indicating more manic symptoms. Some clinical information such as onset age, illness duration, episode times, psychotic symptoms and so on were recorded.

\section{MRI data acquisition and preprocessing}

MRI images were acquired on a 3.0 T Siemens Trio scanner (Siemens, Munich, Germany). During the MRI scanning, foam pads were placed on both sides of subjects' heads to limit head movement. And cotton plugs were used to reduce noise and protect subjects' hearing. Each subject was told to stay awake, keep their eyes closed and not think about anything in particular during the MRI scanning. The subjects were asked after the MRI scanning if they had fallen asleep in the scanner. If a subject did not keep awake during the scan, the data was excluded. T1-weighted images were acquired by employing three-dimensional magnetizationprepared rapid acquisition gradient echo (3D MPRAGE) protocol. Detailed sampling parameters covering the entire brain include repetition time $(\mathrm{TR})=2300 \mathrm{~ms}$, echo time $(\mathrm{TE})=2.03 \mathrm{~ms}$, flip angle $=9^{\circ}$, inversion time $=900 \mathrm{~ms}$, field of view $(\mathrm{FOV})=256 \mathrm{~mm} * 256 \mathrm{~mm}$, matrix $=256 * 256$, thickness $=1 \mathrm{~mm}$ and gap $=0 \mathrm{~mm}$. Resting-state fMRI data were collected by plane echo imaging sequence. The detailed parameters were set as: 30 axial slices, $\mathrm{TR}=2000 \mathrm{~ms}$, $\mathrm{TE}=30 \mathrm{~ms}, \mathrm{FOV}=240 \mathrm{~mm} * 240 \mathrm{~mm}$, gap $=0.4 \mathrm{~mm}$, slice thickness $=4 \mathrm{~mm}$, flip angle $=90^{\circ}$, matrix $=64 * 64$ and scanning time is $500 \mathrm{~s}$.

FreeSurfer 6.0 software (http://surfer.nmr.mgh.harvard. edu/) was used for T1-weighted images processing. Brain volumetric calculation were conducted by recon stream ("recon-all"). The procedure including correction, skull stripped, intensity normalization, automated Talairach transformation, gray/white matter tessellation, and topology correction(Fischl et al., 2001). The segmented subcortical structures were examined by a nonlinear warping atlas, and then the subcortical structure volumes were obtained, including thalamus, caudate, putamen, amygdala, hippocampus, pallidum, and accumbens. Grey matter cortical thickness values were calculated with 68 (34 regions for each hemisphere) cortical regions based on Desikan-Killiany atlas(Desikan et al., 2006). The bilateral thalamus volumes and frontal grey mater cortical thickness of each subject were extracted for further research.

MRI images were preprocessed by Data Processing and Analysis of Brain Imaging (DPABI V5.1,http://rfmri.org/ dpabi Yan et al., 2016). Resting-state fMRI signals were processed in the following steps. To remove the $\mathrm{T} 1$ saturation effect, the first 10 volumes of fMRI images were discarded. The images were then sectioned for slice timing, realignment, nuisance covariates regression (Friston 24-parameter model, white matter signals, cerebrospinal fluid signals and global signals) and spatial normalization $\left(3 * 3 * 3 \mathrm{~mm}^{3}\right)$. Subjects with exceeded head motion (more than $2.0 \mathrm{~mm}$ translation or $2.0^{\circ}$ rotational movements) will be excluded from further research. Data were band-pass filtered, preserving frequencies between 0.01 and $0.08 \mathrm{~Hz}$. The fMRI images were further spatial smoothed using a $6 \mathrm{~mm}$ Gaussian kernel of FWHM. Finally, scrubbing was performed for motion correction to reduce the negative influence in functional connectivity(Power et al., 2012).

\section{Functional connectivity analysis}

Bilateral thalamus from Automated Anatomical Labeling (AAL) parcellation template(Tzourio-Mazoyer et al., 2002) were defined as seeds of regions of interest (ROIs). For each subject, the time series of the bilateral thalamus were extracted, and Pearson correlation coefficients between fMRI signals of the thalamus and that of all voxels of the brain were calculated. These $r$ values were then converted to $\mathrm{z}$ values using Fisher r-to-z transformation to generate a map of functional connectivity. For the purpose of investigating relation between thalamus and frontal cortex, FC maps of the whole brain was masked into frontal cortex with a frontal mask from AAL template. Signals of the brain regions showing significant difference in $\mathrm{FC}$ among the three groups were extracted and further analyzed for post hoc $\mathrm{T}$ test between groups.

\section{Statistical analysis}

Statistical analysis was performed using IBM SPSS software (version 25.0, Armonk, NY, United States). The classification variables (gender, psychotic symptoms, types of bipolar disorder and family history) were tested by Pearson chi-square test. One-way analysis of variance (ANOVA) was used for testing group differences of continuous variables, thalamic volumes and frontal grey 
matter thickness among the three groups (total intracranial volume (TIV) regressed) and two-sample T test was used for post hoc between-group multiple comparison (TIV regressed). Correlations between clinical scores and thalamic volumes/frontal grey matter thickness in the patient groups were calculated with total intracranial volume regressed. Due to the small sample of each group in the current study, effect size (Cohen's d) was calculated to disclose between-group difference of volume/thickness and FC.

Difference of thalamo-frontal FC among mPBD, ePBD patients and HCs was analyzed by ANOVA. Gaussian random field's (GRF) theory was performed for correcting process, with voxel-wised $p<0.005$ and cluster-wised $\mathrm{p}<0.05$. The brain regions showing significant differences among the three groups were identified as ROIs. The average FC value of each ROIs was extracted for subsequent two-sample $\mathrm{T}$ test analysis to compare the between-group FC values difference. Bonferroni correction was used for controlling false positive rate. In PBD group, voxel-wised correlation analysis was conducted between clinical indices and thalamofrontal FC values with age and gender regressed (GRF corrected, with voxel-wised $\mathrm{p}<0.005$ and cluster-wised $\mathrm{p}<0.05)$.

\section{Results}

\section{Demographic and clinical data}

No subject had fallen asleep during the MRI scanning. As displayed in Table 1 , there was no significant differences in age $(p=0.080)$, gender $(p=0.684)$, years of education $(\mathrm{p}=0.259), \mathrm{IQ}(\mathrm{p}=0.085)$ and MFQ scores $(\mathrm{p}=0.738)$ among mPBD group, ePBD group and HCs. The difference of YMRS score $(p<0.001)$ among the three groups was statistically significant. Meanwhile, no significant difference was found between $\mathrm{mPBD}$ and ePBD patients in onset age $(\mathrm{p}=0.410)$, illness duration $(\mathrm{p}=0.065)$, episode times $(\mathrm{p}=0.360)$, psychotic symptoms $(\mathrm{p}=0.342)$, type of bipolar disorder $(\mathrm{p}=0.168)$, and familial $\mathrm{BD}$ history $(\mathrm{p}=0.736)$.

\section{Difference of thalamic volume and frontal cortical thickness among groups}

As shown in Table 2, volume of the left thalamus (THA), grey matter cortical thickness of bilateral opercular inferior frontal gyrus (IFG.ope), middle frontal gyrus (MFG) and superior frontal gyrus(SFG) showed differences among the three groups. Post hoc analysis exhibited that compared with that of the HCs, the volume of left thalamus and cortical thickness of

Table 1 Demographic characteristics and clinical variables

\begin{tabular}{|c|c|c|c|c|c|}
\hline Characteristics & $\operatorname{mPBD}(\mathrm{n}=20)$ & $\operatorname{ePBD}(n=20)$ & $\operatorname{HCs}(\mathrm{n}=19)$ & Statistic $\left(\mathrm{F} / \mathrm{T} / \chi^{2}\right)$ & p-value \\
\hline Gender (male/female) & $8 / 12$ & $10 / 10$ & $7 / 12$ & $0.76^{\mathrm{c}}$ & 0.684 \\
\hline Age (years) & $15.10 \pm 1.65$ & $15.30 \pm 1.72$ & $14.16 \pm 1.57$ & $2.641^{\mathrm{a}}$ & 0.080 \\
\hline Education (years) & $8.35 \pm 1.76$ & $8.40 \pm 1.85$ & $7.47 \pm 2.22$ & $1.385^{\mathrm{a}}$ & 0.259 \\
\hline IQ & $100.10 \pm 13.56$ & $107.50 \pm 9.62$ & $105.32 \pm 7.51$ & $2.579^{\mathrm{a}}$ & 0.085 \\
\hline YMRS scores & $34.60 \pm 6.32$ & $5.35 \pm 1.60$ & $3.63 \pm 2.06$ & $380.3^{\mathrm{a}}$ & $<0.001$ \\
\hline MFQ scores & $7.20 \pm 2.59$ & $6.60 \pm 4.44$ & $6.37 \pm 3.00$ & $0.305^{\mathrm{a}}$ & 0.738 \\
\hline Onset age (years) & $14.00 \pm 1.69$ & $13.50 \pm 2.09$ & & $0.833^{\mathrm{b}}$ & 0.410 \\
\hline Illness duration (months) & $13.65 \pm 11.46$ & $22.55 \pm 17.56$ & & $-1.898^{b}$ & 0.065 \\
\hline Episode times & $3.05 \pm 1.70$ & $4.65 \pm 7.53$ & & $-0.926^{b}$ & 0.360 \\
\hline Psychotic symptoms (yes/no) & $9 / 11$ & $12 / 8$ & & $0.902^{\mathrm{c}}$ & 0.342 \\
\hline BD-I/BD-II & $16 / 4$ & $12 / 8$ & & $1.905^{\mathrm{c}}$ & 0.168 \\
\hline Familial BD history (yes/no) & $7 / 13$ & $6 / 14$ & & $0.114^{\mathrm{c}}$ & 0.736 \\
\hline \multicolumn{6}{|l|}{ Medications } \\
\hline Lithium(n/\%) & $9 / 45 \%$ & $7 / 35 \%$ & & & \\
\hline Valproate(n/\%) & $11 / 55 \%$ & $15 / 75 \%$ & & & \\
\hline Atypical antipsychotics(n/\%) & $14 / 70 \%$ & $17 / 85 \%$ & & & \\
\hline Antidepressants(n/\%) & $1 / 5 \%$ & & & & \\
\hline
\end{tabular}

${ }^{\mathrm{a}}$ one-way ANOVA; ${ }^{\mathrm{b}}$ Two-sample t-test; ${ }^{\mathrm{c}}$ chi-square test.

Values are presented as mean \pm standard deviation.

mPBD: manic pediatric bipolar disorder; ePBD: euthymic pediatric bipolar disorder; HCs: healthy controls; IQ: intelligence quotient; YMRS: Young Manic Rating Scale; MFQ: Mood and Feelings Questionnaire; BD-I, bipolar disorder type I; BD-II, bipolar disorder type II. 
Table 2 Regions showing differences in thalamic volume and frontal grey matter thickness among mPBD, ePBD patients and HCs

\begin{tabular}{|c|c|c|c|c|c|c|c|c|c|c|c|}
\hline \multirow[t]{3}{*}{ Region } & \multicolumn{2}{|c|}{ Main effect } & \multirow{2}{*}{\multicolumn{2}{|c|}{ mPBD vs. ePBD }} & \multicolumn{3}{|l|}{ Post Hoc } & \multirow{2}{*}{\multicolumn{4}{|c|}{ ePBD vs. HCs }} \\
\hline & \multirow[b]{2}{*}{$\mathrm{F}$} & \multirow[b]{2}{*}{$\mathrm{p}$} & & & \multicolumn{3}{|c|}{ mPBD vs. $\mathrm{HCs}$} & & & & \\
\hline & & & $\mathrm{T}$ & $\mathrm{p}$ & Cohen'd & $\mathrm{T}$ & $\mathrm{p}$ & Cohen'd & $\mathrm{T}$ & $\mathrm{p}$ & Cohen'd \\
\hline THA.L & 6.184 & $0.004 * *$ & -1.790 & 0.237 & -0.589 & -3.516 & $0.003 * *$ & -1.044 & -1.749 & 0.257 & -0.585 \\
\hline THA.R & 2.474 & 0.093 & & & & & & & & & \\
\hline IFG.ope.L & 3.934 & $0.025^{*}$ & -0.062 & 1.000 & -0.02 & & & & & & \\
\hline IFG.orb.L & 2.569 & 0.086 & & & & & & & & & \\
\hline IFG.tri.L & 3.091 & 0.053 & & & & & & & & & \\
\hline MFG.L & 4.906 & $0.011^{*}$ & -0.37 & 1.000 & -0.12 & -2.892 & $0.016^{*}$ & -0.95 & -2.517 & $0.044 *$ & -0.83 \\
\hline SFG.L & 6.585 & $0.003 * *$ & -0.005 & 1.000 & -0.001 & -3.159 & $0.008 * *$ & -1.04 & -3.153 & $0.008 * *$ & -1.04 \\
\hline PreCG.L & 2.441 & 0.096 & & & & & & & & & \\
\hline IFG.ope.R & 3.691 & $0.031 *$ & -0.939 & 1.000 & -0.30 & -2.681 & $0.029 *$ & -0.88 & -1.754 & 0.255 & -0.57 \\
\hline IFG.orb.R & 0.405 & 0.669 & & & & & & & & & \\
\hline IFG.tri.R & 2.083 & 0.134 & & & & & & & & & \\
\hline MFG.R & 3.555 & $0.035^{*}$ & -1.280 & 0.617 & -0.42 & -2.666 & $0.030 *$ & -0.87 & -1.401 & 0.499 & -0.46 \\
\hline SFG.R & 4.264 & $0.019 *$ & -0.128 & 1.000 & -0.04 & -2.601 & $0.036^{*}$ & -0.85 & -2.473 & $0.049 *$ & -0.81 \\
\hline PreCG.L & 2.131 & 0.128 & & & & & & & & & \\
\hline
\end{tabular}

mPBD: manic pediatric bipolar disorder; ePBD: euthymic pediatric bipolar disorder; HCs: healthy controls; THA.L: left thalamus; THA.R: right thalamus.

Presented adjusted $\mathrm{p}<0.05$ was considered to indicate a significant difference (Bonferroni corrected).

$* * \mathrm{p}<0.01, * \mathrm{p}<0.05$.

IFG.ope: opercular inferior frontal gyrus; IFG.orb: orbital inferior frontal gyrus; IFG.tri: triangular inferior frontal gyrus; MFG: middle frontal gyrus; SFG: superior frontal gyrus.

right MFG significantly decreased in $\mathrm{MPBD}$ patients, cortical thickness of left MFG, left SFG and right SFG was significantly decreased in both mPBD and ePBD patients $(\mathrm{p}<0.05$, Bonferroni corrected).

\section{Differences in thalamo-frontal FC}

As a result of ANOVA on resting-state FC of mPBD, ePBD and $\mathrm{HCs}$ groups, there were significant differences in $\mathrm{FC}$ between left thalamus and right MFG, left precentral gyrus (PreCG) and right SFG, and FC between the right thalamus and right MFG(Fig. 1). These brain regions were detailed in Table 3. The fMRI signals of the above areas were extracted for further post hoc tests. As shown in Fig. 2, in mPBD group, FC between left THA and right MFG (BA9, BA11) was reduced compared with that of the $\mathrm{HCs}$ (Fig. 2A,2D), and FC between left THA and right MFG(BA9) was reduced compared with that of the ePBD subjects (Fig. 2E). In ePBD group, FC between left THA and left PreCG, and right SFG were significantly reduced compared with that of the HCs (Fig. 2B,2C) $(\mathrm{p}<0.05$, Bonferroni corrected).

\section{Correlation analysis between clinical indices and FC in PBD}

In the present study, no significant correlation was found between the clinical indices and thalamic volume and frontal grey matter thickness in bipolar disorder patients. Voxel wised correlation analysis was conducted between clinical indices and thalamo-frontal FC values, and the significant results were exhibited in Fig. 3. In ePBD patients, episode times positively correlated with $\mathrm{FC}$ between left THA and right PreCG(BA6) (Fig. 3A,3C) and FC between right THA and right PreCG(BA6) (Fig. 3B,3D). (GRF corrected, voxel $\mathrm{p}<0.005$, cluster $\mathrm{p}<0.05)$.

\section{Discussion}

The goal of the present study is to explore alterations in volume of the thalamus, cortical thickness of the frontal regions and thalamo-frontal $\mathrm{FC}$ by analyzing $\mathrm{T} 1$ and BOLD signals of manic PBD, euthymic PBD and HCs. Several 
Fig. 1 Statistical difference maps showing difference in thalamo-frontal FC among mPBD, ePBD patients and HCs by ANOVA analysis. $(\mathrm{p}<0.05$, GRF corrected). THA.L: left thalamus; THA.R: right thalamus; MFG: middle frontal gyrus; SFG: superior frontal gyrus; PreCG: precentral gyrus

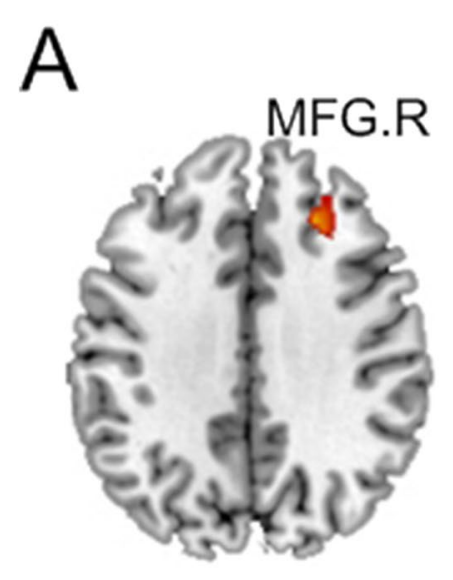

Seed: THA.L

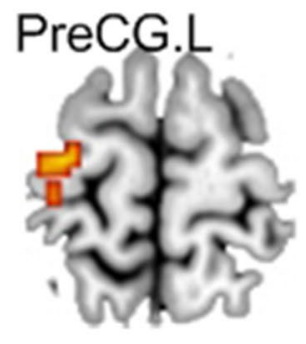

$Z=69$

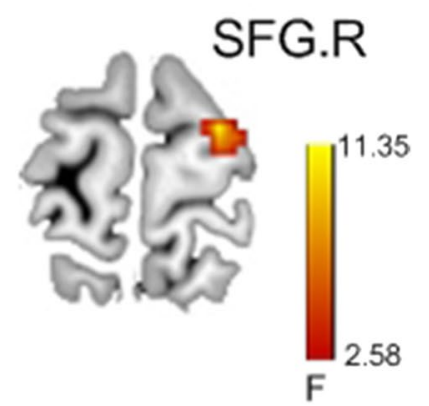

$Z=72$

\section{B}

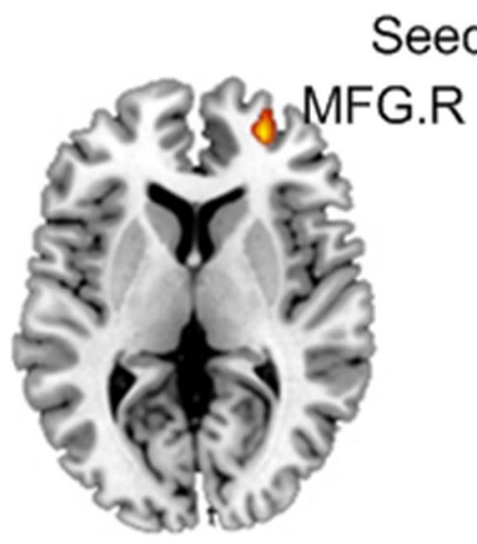

$Z=6$

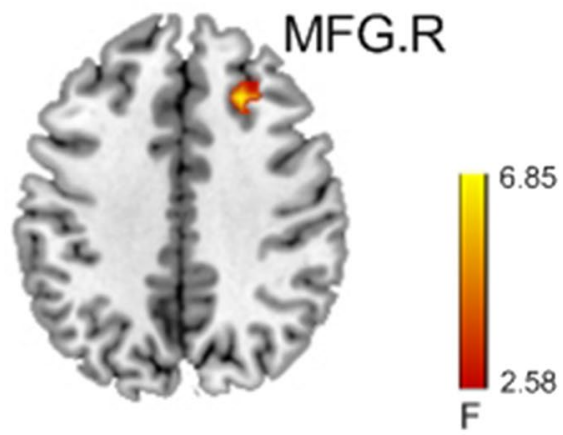

$Z=39$

Table 3 Regions showing difference in thalamo-frontal FC among mPBD, ePBD patients and HCs

\begin{tabular}{lllll}
\hline & $\begin{array}{l}\text { Brodmann } \\
\text { Area }\end{array}$ & $\begin{array}{l}\text { MNI Coordinates } \\
\text { X Y Z }\end{array}$ & $\begin{array}{l}\text { Peak F } \\
\text { value }\end{array}$ & Cluster size \\
\hline Seed:THA.L & & & & \\
MFG.R & 9 & 253037 & 8.63 & 71 \\
SFG.R & 6 & $24-972$ & 8.17 & 62 \\
PreCG.L & 6 & $-30-1572$ & 5.32 & 63 \\
Seed:THA.R & & & & \\
MFG.R & 11 & 27516 & 6.78 & 57 \\
MFG.R & 9 & 242739 & 6.27 & 58 \\
\hline
\end{tabular}

mPBD: manic pediatric bipolar disorder; ePBD: euthymic pediatric bipolar disorder; HCs: healthy controls; L: left; R:right; MNI, Montreal Neurological Institute; SFG: superior frontal gyrus; MFG: middle frontal gyrus; PreCG: precentral gyrus.

observations were obtained. First, both mPBD and ePBD subjects exhibited reduction in grey matter cortical thickness in the two groups include left MFG and bilateral SFG. While decreased volume of left thalamus and decreased cortical thickness of right IFG.ope and right MFG were only found in $\mathrm{mPBD}$. Second, FC between the bilateral thalamus and MFG significantly decreased in mPBD patients compared with that of the HCs and ePBD subjects. FC between thalamus and SFG/PreCG was increased in ePBD compared to HCs. Third, in ePBD group, episode times was significantly positively correlated with the FC between thalamus and SFG/PreCG. These findings provide new evidence for shared and specific structural and functional connectivity disruptions, which may help to disclose neuropathological mechanisms underlying subtypes of PBD.

We found volume reduction in the left thalamus and gray matter cortical thickness reduction of frontal cortex in PBD subjects. It had been indicated that frontal-subcortical areas was early involved in BD development(Sigitova et al., 2017). The thalamus serves as a relay station within forebrain circuits to the cerebral cortex and to limbic structures(Jones, 1997). Significantly lower bilateral thalamic volumes in BD (Hibar et al., 2016) was demonstrated. No thalamic volume alteration were detected in ePBD subjects in the present study, which consisting 

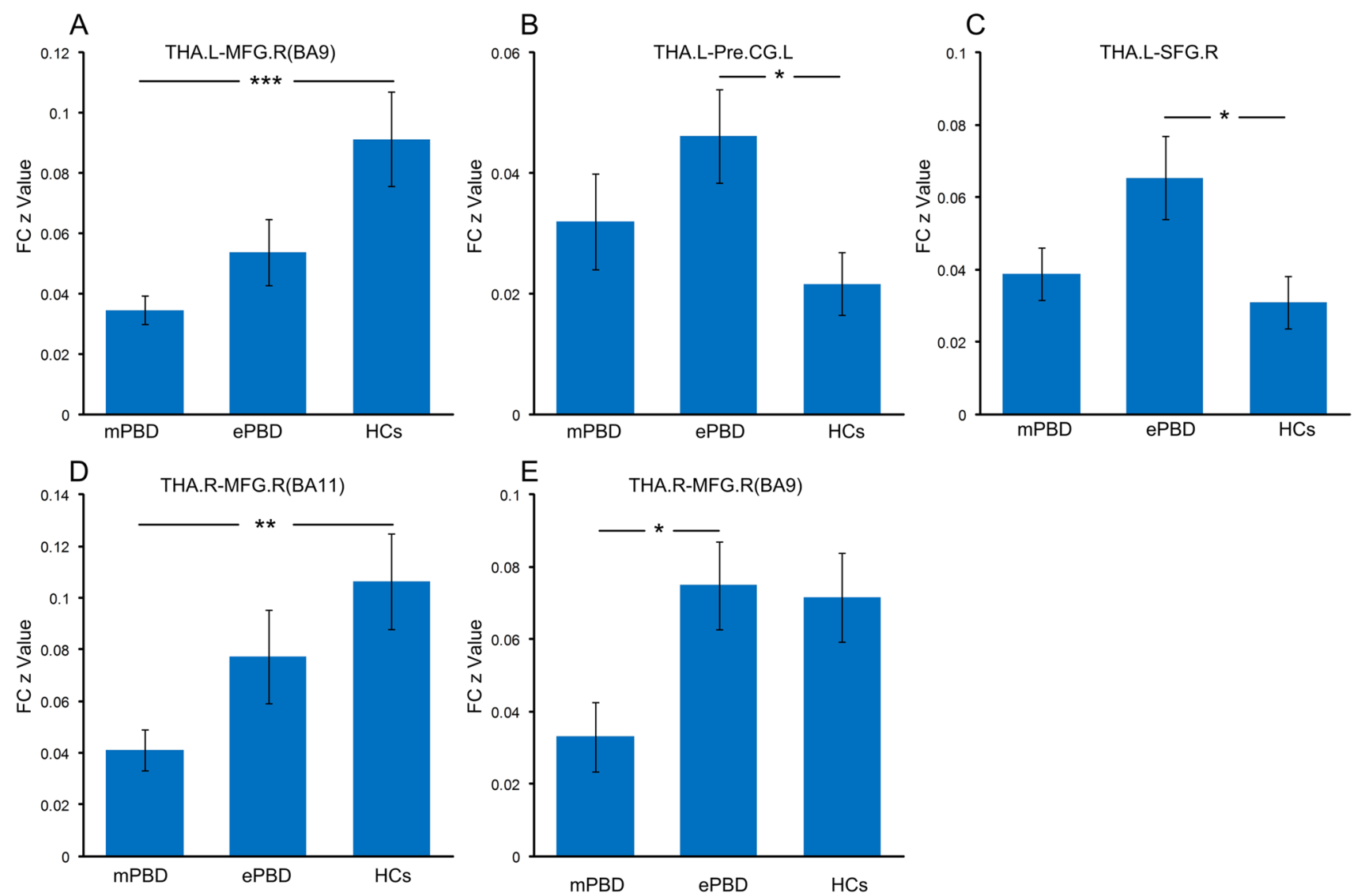

Fig. 2 Pair wise comparison of $\mathrm{z}$ values of thalamo-frontal $\mathrm{FC}$ in mPBD, ePBD and HCs groups. (Two-sample T test; $p<0.05$, Bonferroni corrected) mPBD: manic pediatric bipolar disorder; ePBD: euthymic pediatric bipolar disorder; HCs: healthy controls; L: left;

R: right; SFG: superior frontal gyrus; MFG: middle frontal gyrus; PreCG: precentral gyrus; BA: Brodmann area *: $\mathrm{p}<0.05$; **: $\mathrm{p}<0.01 ; * * *: \mathrm{p}<0.001$
Fig. 3 Voxel-wised correlation analysis between episode times and thalamo-frontal FC values in euthymic PBD. Episode times positively correlated with FC between THA.L and PreCG.R(A, C) and FC between THA.R and PreCG.R(B, D) (GRF corrected, voxel $\mathrm{p}<0.005$, cluster $\mathrm{p}<0.05)$ Asterisk (*) represents the adjusted values with age and gender regressed. PreCG: precentral gyrus; L: left; R: right
Seed: THA.L

A

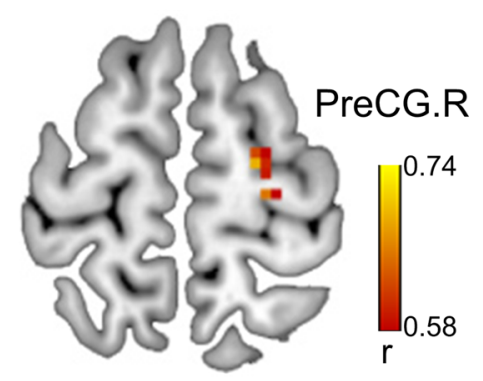

$Z=65$

C

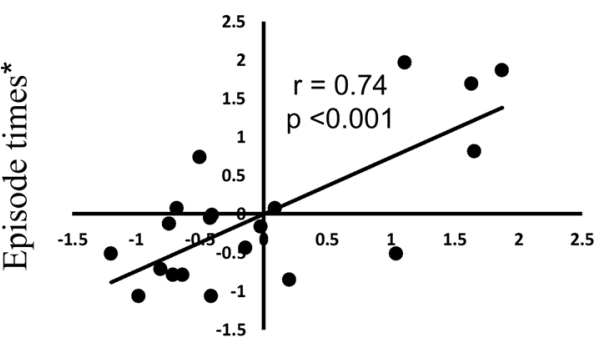

THA.L-PreCG.R FC*
Seed: THA.R

B

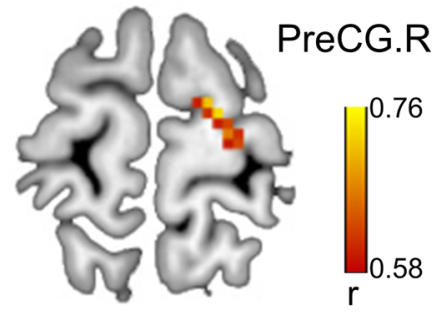

$Z=70$

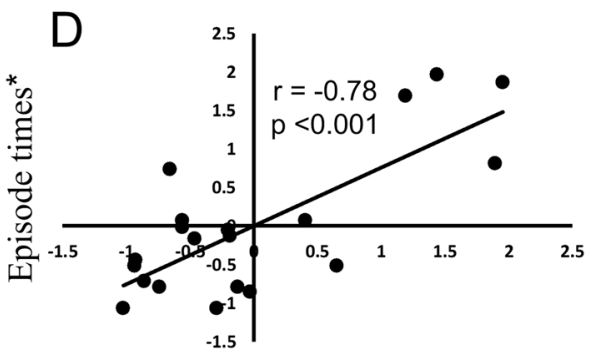

THA.R-PreCG.R FC* 
with prior results(Radenbach et al., 2010). The prefrontal cortex plays a key role in "top-down" inhibitory control of internal and external sensory driven compulsive behaviors. Some studies had reported grey matter reduction of the prefrontal cortex(Soloff et al., 2008; Yu et al., 2019), which may contribute to the dysregulation of affect and emotion in BD(Soloff et al., 2008). The grey matter of the human cerebral cortex is made up of neurons and glial cells. Neurotrophins, which was found to be related with formation of new neurons or glial cells in animal models, was confirmed to decrease in manic and depressive of BD(Duman \& Monteggia, 2006). Meanwhile, significantly reduced concentrations of phosphocreatine in grey matter in manic and euthymic bipolar I disorder subjects had also been reported. Phosphocreatine is accounted for decreased neuronal cell density or impaired oxidative phosphorylation(Dudley et al., 2016). Taken together, these factors may play an important role in brain cell development and may induce abnormal development of a brain area, which may cause the grey matter volume reduction of the thalamus and frontal cortex in PBD. Reduction of cortical thickness of the same prefrontal regions in the mPBD and ePBD subjects may suggest that dysfunction of the prefrontal cortex persist across different mood states.

In the present study, there is no shared alteration of thalamo-frontal connections in the MPBD and ePBD subjects. In manic PBD, aberrant thalamo-prefrontal connections was characterized by decreased thalamo-prefrontal FC(PFC) with right MFG (BA9, BA11) compared to HCs, and with right MFG(BA9) compared to ePBD. The results are consistent with previous study showing thalamo-PFC under-connectivity in bipolar disorder subjects(Anticevic et al., 2014). In a study combining schizophrenia and bipolar I disorder, reduced PFC-thalamic connectivity was confirmed in both chronic and early-stage psychosis patients(Woodward \& Heckers, 2016). In addition, circuits in corticolimbic model(Brooks \& Vizueta, 2014), was contributed to some clinical features of bipolar disorder(Strakowski et al., 2012). PFC areas in these circuits, including dorsolateral PFC(DLPFC)(BA9) and ventromedial PFC(VMPFC) (BA11), both have reciprocal projections with the thalamus. The DLPFC, playing an essential role in mood regulation, decision making and working memory, exhibited decreased metabolism in mania(Gonul et al., 2009). The MPFC is related to self-referential thought and reviewing past knowledge for preparing future action. In a mouse experiment, interaction between mediodorsal thalamus and MPFC was verified to play a key role in supporting working memory maintenance(Bolkan et al., 2017). The current findings of reduction of thalamic FC with DLPFC and VMPFC in mPBD may indicate a deficit in bottom-up attention and emotion processing, and the thalamofrontal feedback loops may be an important pathophysiological marker in PBD.
In euthymic PBD of the present study, thalamo-frontal connections with left PreCG (BA6) and right SFG(BA6) were increased compared with that of HCs. Meanwhile, episode times of historical manic or depressive symptom positively correlated with the FC values between bilateral thalamus and right left PreCG (BA6). The precentral gyrus is on the lateral surface of each frontal lobe, and it is the location of primary motor cortex which accounts for voluntary motor movement controlling. As a complicated sensory information node, the thalamus receives and processes sensory and motor input signals. The thalamus and precentral gyrus were reported to exhibit abnormality in ePBD patients. During emotion labeling condition, right thalamus showed decreased activation in remitted BD patients(Foland-Ross et al., 2012). Relative to healthy controls, remitted BD subjects demonstrated increased activation of PreCG in twoback task(Monks et al., 2004). Meanwhile, thalamocortical hyperconnectivity in sensory regions including motor visual, and occipital cortex has been characterized in BD (Ramsay, 2019). Hyperconnectivity between thalamus and precentral gyrus(BA6) was found in early stage of psychosis including schizophrenia and BD, which was interpreted as refinement of somatomotor-thalamic connectivity(Woodward \& Heckers, 2016). Remitted BD subjects was verified showing increased thalamic connectivity with bilateral sensory-motor cortices, which may reflecting sensory gating disruption(Anticevic et al., 2014). Thalamic over-connectivity with primary motor cortex in ePBD in the present study is consistent with the above prior studies. Thalamo-primary motor cortex disturbances was only found in euthymic PBD, not in manic PBD subjects, which seems that the observed disturbances might somewhat be stable and be not altered or worsen during acute psychosis. However, the positively correlation between the thalamic-primary motor cortex connectivity and historical manic or depressive episode times may suggest the altered connectivity between thalamus and primary motor cortex also associated with historical symptom exacerbation of PBD.

\section{Limitations}

The present study also has several limitations. Firstly, the small sample size may not large enough to detect volumetric or FC differences among groups completely, more data will be collected in future work to make the research more convinced. Secondly, all PBD patients in the current study received medication therapy, which may influence on interpreting the present result. Although researches have shown no effect of medication in neural differences between patient and controls(Jiang et al., 2020), future studies need to verify findings of the current study in patients without medication. Thirdly, the FC was investigated between the 
whole thalamus and all of the voxels of the frontal cortex. The approach provides better specificity within the frontal cortex but may obscure probing specific abnormalities of the nuclei of the thalamus. Actually studies had indicated a fatal role of mediodorsal thalamus in sustaining prefrontal activity during working memory maintenance(Bolkan et al., 2017) and altered mediodorsal thalamus activity may disrupt prefrontal-dependent cognitive behaviors(Parnaudeau et al., 2013). Future work would investigate structural and functional alterations of sub-nuclei of thalamus in PBD rather treating the thalamus as a homogeneous structure.

\section{Conclusions}

The findings of the present study demonstrated detailed knowledge regarding shared and specific structural and functional alterations in thalamo-frontal loop in manic PBD and euthymic PBD subjects. The thalamo-frontal abnormalities reported in adult BD subjects are also observed in adolescent patients, and thalamo-frontal dysfunction may be a crucial treatment target in BD.

Acknowledgements The authors thank all participants as well as investigators involved in the present study.

\begin{abstract}
Author contributions Author contributions included conception and study design (Qing Jiao and Guang-Ming Lu), data collection or acquisition and clinical support (Wei-Jia Gao and Lin-Yan Su), statistical analysis (Yi-Bing Guo, Zhi-Liang Long and Dong Cui), interpretation of results(Yong-Xin Guo and Jian-Feng Qiu), drafting the manuscript work or revising it critically for important intellectual content (Yi-Bing Guo and Wei-Fang Cao) and approval of final version to be published and agreement to be accountable for the integrity and accuracy of all aspects of the work (All authors).
\end{abstract}

Funding The present work was supported by the Funds for the National Natural Science Foundation of China (81371531 to Qing Jiao; 81901725 to Zhi-Liang Long; 81901730 to Wei-Fang Cao), Natural Science Foundation of Zhejiang Province(LQ19H090018 to Wei-Jia Gao), High-level cultivation program of Shandong First Medical University \&Shandong Academy of Medical Sciences (2017GCC11 to Wei-Fang Cao) and Taishan Scholars Program of Shandong Province (TS201712065 to Jian-Feng Qiu), Academic promotion programme of Shandong First Medical University (2019QL009 to Jian-Feng Qiu).

All procedures performed in studies involving human participants were in accordance with the ethical standards of the institutional and national research committee and with the 1964 Helsinki declaration and its later amendments or comparable ethical standards. Informed consent was obtained from all individual participants included in the present study.

\section{Declarations}

Conflict of interest All authors confirm that they have read the journal's position on issues involved in ethical publication and have no conflict of interests.
Open Access This article is licensed under a Creative Commons Attribution 4.0 International License, which permits use, sharing, adaptation, distribution and reproduction in any medium or format, as long as you give appropriate credit to the original author(s) and the source, provide a link to the Creative Commons licence, and indicate if changes were made. The images or other third party material in this article are included in the article's Creative Commons licence, unless indicated otherwise in a credit line to the material. If material is not included in the article's Creative Commons licence and your intended use is not permitted by statutory regulation or exceeds the permitted use, you will need to obtain permission directly from the copyright holder. To view a copy of this licence, visit http://creativecommons.org/licenses/by/4.0/.

\section{References}

Altinay, M. I., Hulvershorn, L. A., Karne, H., Beall, E. B., \& Anand, A. (2016). Differential Resting-State Functional Connectivity of Striatal Subregions in Bipolar Depression and Hypomania. Brain Connect, 6(3), 255-265. https://doi.org/10.1089/brain. 2015.0396

Anticevic, A., Cole, M. W., Repovs, G., Murray, J. D., Brumbaugh, M. S., Winkler, A. M., . . Glahn, D. C. (2014). Characterizing thalamo-cortical disturbances in schizophrenia and bipolar illness. Cereb Cortex, 24(12), 3116-3130. https://doi.org/10.1093/ cercor/bht 165

Arumugham, S. S., Torres, I. J., Lang, D. J., Su, W., Lam, R. W., Honer, W. G., \& Yatham, L. N. (2017). Subcortical structural volumes in recently remitted first episode mania. Journal of Affective Disorders, 222, 23-27. https://doi.org/10.1016/j.jad. 2017.06.051

Bolkan, S. S., Stujenske, J. M., Parnaudeau, S., Spellman, T. J., Rauffenbart, C., Abbas, A. I., . . Kellendonk, C. (2017). Thalamic projections sustain prefrontal activity during working memory maintenance. Nat Neurosci, 20(7), 987-996. https:// doi.org/10.1038/nn.4568

Brooks, J. O., \& Vizueta, N. (2014). Diagnostic and clinical implications of functional neuroimaging in bipolar disorder. Journal of Psychiatric Research, 57, 12-25. https://doi.org/10.1016/j. jpsychires.2014.05.018

Chang, K., Barnea-Goraly, N., Karchemskiy, A., Simeonova, D. I., Barnes, P., Ketter, T., \& Reiss, A. L. (2005). Cortical magnetic resonance imaging findings in familial pediatric bipolar disorder. Biological Psychiatry, 58(3), 197-203. https://doi.org/10. 1016/j.biopsych.2005.03.039

Chen, L., Wang, Y., Niu, C., Zhong, S., Hu, H., Chen, P., . . Huang, R. (2018). Common and distinct abnormal frontal-limbic system structural and functional patterns in patients with major depression and bipolar disorder. Neuroimage Clin, 20, 42-50. https:// doi.org/10.1016/j.nicl.2018.07.002

Chen, Z., Cui, L., Li, M., Jiang, L., Deng, W., Ma, X., . . Li, T. (2012). Voxel based morphometric and diffusion tensor imaging analysis in male bipolar patients with first-episode mania. Prog Neuropsychopharmacol Biol Psychiatry, 36(2), 231-238. https:// doi.org/10.1016/j.pnpbp.2011.11.002

Desikan, R. S., Segonne, F., Fischl, B., Quinn, B. T., Dickerson, B. C., Blacker, D., . . Killiany, R. J. (2006). An automated labeling system for subdividing the human cerebral cortex on MRI scans into gyral based regions of interest. Neuroimage, 31(3), 968-980. https://doi.org/10.1016/j.neuroimage.2006.01.021

Dudley, J., DelBello, M. P., Weber, W. A., Adler, C. M., Strakowski, S. M., \& Lee, J. H. (2016). Tissue-dependent cerebral energy metabolism in adolescents with bipolar disorder. Journal of Affective Disorders, 191, 248-255. https://doi.org/10.1016/j. jad.2015.11.045 
Duman, R. S., \& Monteggia, L. M. (2006). A neurotrophic model for stress-related mood disorders. Biological Psychiatry, 59(12), 1116-1127. https://doi.org/10.1016/j.biopsych.2006.02.013

Fischl, B., Liu, A., \& Dale, A. M. (2001). Automated manifold surgery: Constructing geometrically accurate and topologically correct models of the human cerebral cortex. IEEE Transactions on Medical Imaging, 20(1), 70-80. https://doi.org/10.1109/42.906426

Foland-Ross, L. C., Bookheimer, S. Y., Lieberman, M. D., Sugar, C. A., Townsend, J. D., Fischer, J., . . Altshuler, L. L. (2012). Normal amygdala activation but deficient ventrolateral prefrontal activation in adults with bipolar disorder during euthymia. Neuroimage, 59(1), 738-744. https://doi.org/10.1016/j.neuroimage.2011.07.054

Frazier, J. A., Chiu, S., Breeze, J. L., Makris, N., Lange, N., Kennedy, D. N., . . Biederman, J. (2005). Structural brain magnetic resonance imaging of limbic and thalamic volumes in pediatric bipolar disorder. Am J Psychiatry, 162(7), 1256-1265. https:// doi.org/10.1176/appi.ajp.162.7.1256

Goldstein, B. I., \& and Boris Birmaher. (2012). Prevalence, Clinical Presentation and Differential Diagnosis of Pediatric Bipolar Disorder. The Israel Journal of Psychiatry and Related Sciences, 49(1), 3-14.

Gonul, A. S., Coburn, K., \& Kula, M. (2009). Cerebral blood flow, metabolic, receptor, and transporter changes in bipolar disorder: The role of PET and SPECT studies. International Review of Psychiatry, 21(4), 323-335. https://doi.org/10.1080/09540260902962131

Hajek, T., Cullis, J., Novak, T., Kopecek, M., Blagdon, R., Propper, L., ... Alda, M. (2013). Brain structural signature of familial predisposition for bipolar disorder: replicable evidence for involvement of the right inferior frontal gyrus. Biol Psychiatry, 73(2), 144-152. https://doi.org/10.1016/j.biopsych.2012.06.015

Hibar, D. P., Westlye, L. T., Doan, N. T., Jahanshad, N., Cheung, J. W., Ching, C. R. K., . . . Andreassen, O. A. (2018). Cortical abnormalities in bipolar disorder: an MRI analysis of 6503 individuals from the ENIGMA Bipolar Disorder Working Group. Mol Psychiatry, 23(4), 932-942. https://doi.org/10.1038/mp.2017.73

Hibar, D. P., Westlye, L. T., van Erp, T. G., Rasmussen, J., Leonardo, C. D., Faskowitz, J., . . . Andreassen, O. A. (2016). Subcortical volumetric abnormalities in bipolar disorder. Mol Psychiatry, 21(12), 1710-1716. https://doi.org/10.1038/mp.2015.227

Jiang, X., Fu, S., Yin, Z., Kang, J., Wang, X., Zhou, Y., . . Tang, Y. (2020). Common and distinct neural activities in frontoparietal network in first-episode bipolar disorder and major depressive disorder: Preliminary findings from a follow-up resting state fMRI study. J Affect Disord, 260, 653-659. https://doi.org/10.1016/j. jad.2019.09.063

Jones, E. G. (1997). Cortical development and thalamic pathology in schizophrenia. Schizophrenia Bulletin, 23(3), 483-501. https:// doi.org/10.1093/schbul/23.3.483

Kaufman, J., \& B. B., Brent D, Rao U, Flynn C, Moreci P, Williamson D, Ryan N. . (1997). Schedule for Affective Disorders and Schizophrenia for School-Age Children-Present and Lifetime Version (K-SADS-PL): Initial reliability and validity data. Journal of the American Academy of Child and Adolescent Psychiatry, 36(7), 9. https://doi.org/10.1097/00004583-199707000-00021

Liberg, B., Ekman, C. J., Sellgren, C., Johansson, A. G., \& Landen, M. (2015). Subcortical morphometry and psychomotor function in euthymic bipolar disorder with a history of psychosis. Brain Imaging and Behavior, 9(2), 333-341. https://doi.org/10.1007/ s11682-014-9313-0

Monks, P. J., Thompson, J. M., Bullmore, E. T., Suckling, J., Brammer, M. J., Williams, S. C., . . Curtis, V. A. (2004). A functional MRI study of working memory task in euthymic bipolar disorder: evidence for task-specific dysfunction. Bipolar Disord, 6(6), 550-564. https://doi.org/10.1111/j.1399-5618.2004.00147.x

Nery, F. G., Monkul, E. S., \& Lafer, B. (2013). Gray matter abnormalities as brain structural vulnerability factors for bipolar disorder: A review of neuroimaging studiesof individuals at high genetic risk for bipolar disorder. Aust N Z J Psychiatry, 47(12), 1124-1135. https://doi.org/10.1177/0004867413496482

Power, J. D., Barnes, K. A., Snyder, A. Z., Schlaggar, B. L., \& Petersen, S. E. (2012). Spurious but systematic correlations in functional connectivity MRI networks arise from subject motion. NeuroImage, 59(3), 2142-2154. https://doi.org/10.1016/j.neuroimage. 2011.10.018

Radenbach, K., Flaig, V., Schneider-Axmann, T., Usher, J., Reith, W., Falkai, P., . . . Scherk, H. (2010). Thalamic volumes in patients with bipolar disorder. Eur Arch Psychiatry Clin Neurosci, 260(8), 601-607. https://doi.org/10.1007/s00406-010-0100-7

Ramsay, I. S. (2019). An Activation Likelihood Estimate Meta-analysis of Thalamocortical Dysconnectivity in Psychosis. Biol Psychiatry Cogn Neurosci Neuroimaging, 4(10), 859-869. https://doi.org/10. 1016/j.bpsc.2019.04.007

Sigitova, E., Fisar, Z., Hroudova, J., Cikankova, T., \& Raboch, J. (2017). Biological hypotheses and biomarkers of bipolar disorder. Psychiatry and Clinical Neurosciences, 71(2), 77-103. https://doi. org/10.1111/pcn.12476

Soloff, P., Nutche, J., Goradia, D., \& Diwadkar, V. (2008). Structural brain abnormalities in borderline personality disorder: A voxelbased morphometry study. Psychiatry Research, 164(3), 223-236. https://doi.org/10.1016/j.pscychresns.2008.02.003

Son, Y. D., Han, D. H., Kim, S. M., Min, K. J., \& Renshaw, P. F. (2017). A functional connectivity comparison between attention deficit hyperactivity disorder and bipolar disorder in medicationnaive adolescents with mood fluctuation and attention problems. Psychiatry Res Neuroimaging, 263, 1-7. https://doi.org/10.1016/j. pscychresns.2017.02.006

Strakowski, S. M., Adler, C. M., Almeida, J., Altshuler, L. L., Blumberg, H. P., Chang, K. D., . . Townsend, J. D. (2012). The functional neuroanatomy of bipolar disorder: a consensus model. Bipolar Disord, 14(4), 313-325. https://doi.org/10.1111/j.1399. 5618.2012.01022.x

Tzourio-Mazoyer, N., Landeau, B., Papathanassiou, D., Crivello, F., Etard, O., Delcroix, N., . . Joliot, M. (2002). Automated anatomical labeling of activations in SPM using a macroscopic anatomical parcellation of the MNI MRI single-subject brain. Neuroimage, 15(1), 273-289. https://doi.org/10.1006/nimg.2001.0978

Van Meter, A., Moreira, A. L. R., \& Youngstrom, E. (2019). Updated Meta-Analysis of Epidemiologic Studies of Pediatric Bipolar Disorder. J Clin Psychiatry, 80(3). https://doi.org/10.4088/JCP. $18 \mathrm{r} 12180$

Woodward, N. D., \& Heckers, S. (2016). Mapping Thalamocortical Functional Connectivity in Chronic and Early Stages of Psychotic Disorders. Biological Psychiatry, 79(12), 1016-1025. https://doi. org/10.1016/j.biopsych.2015.06.026

Yan, C. G., Wang, X. D., Zuo, X. N., \& Zang, Y. F. (2016). DPABI: Data Processing \& Analysis for (Resting-State) Brain Imaging. Neuroinformatics, 14(3), 339-351. https://doi.org/10.1007/ s12021-016-9299-4

Young, R. C., \& B. J., Ziegler VE, Meyer DA. . (1978). A rating scale for mania reliability, validity and sensitivity. British Journal of Psychiatry, 133, 7. https://doi.org/10.1192/bjp.133.5.429

Yu, H., Meng, Y. J., Li, X. J., Zhang, C., Liang, S., Li, M. L., . . Li, T. (2019). Common and distinct patterns of grey matter alterations in borderline personality disorder and bipolar disorder: voxel-based meta-analysis. Br J Psychiatry, 215(1), 395-403. https://doi.org/ 10.1192/bjp.2019.44

Publisher's Note Springer Nature remains neutral with regard to jurisdictional claims in published maps and institutional affiliations. 